\section{CHRONIC SUPPURATIVE OTITIS MEDIA} ITS OPERATIVE TREATMENT

BY

\author{
M. R. SHERIDAN, M.B., F.R.C.S.ED., D.L.O. \\ CLINICAL ASSISTANT, LIVERPOOL EYE, EAR, AND THROAT \\ INFIRMARY
}

The reasonable man adapts himself to the world; the unreasonable man persists in trying to adapt the world to himself. Therefore all progress depends on the unreasonable man.-G. B. SHAw, "A Revolutionist's Handbook."

A vast number of mastoid operations for chronic suppurative otitis media performed with the threefold purpose of removing dangerous disease, preserving the hearing, and healing the ear achieve their object. A proportion of these operations fail in part, and patients are disgruntled to find that their ears flow as before. I have examined a number of radical mastoid and modified radical mastoid cavities from various sources to try to fathom the causes of failure and thus evolve a technique which would avoid them.

\section{Radical Mastoid Operation}

The observations arrived at after the study of a series of cases are enumerated below. There follows a description of a method of operating which gives a high proportion of dry ears.

1. Many incisions were too short and incorrectly curved. Insufficient exposure hampers good surgery.

2. The bony meatus in some cases was so narrow that proper supervision of the operation cavity must have been impossible.

3. The antrum remained as a recess in the hindmost part of the cavity, filled with granulations, and not epithelialized, in certain cases.

4. Occasionally the bridge had been removed too timidly. Its piers remained untrimmed, leaving the aditus as a gutter between them; over such a surface healing could not possibly take place.

5. In some a prominent facial ridge remained, damming pus and granulations in the mastoid cavity behind it.

6. The existence of a Eustachian fistula spoiled a large number of well-performed radical operations.

7. A high elevation on the bony meatal floor left a hypotympanic trough, difficult to reach in the aftertreatment in some of the cases.

8. Stenosis of some of the cavities had occurred due to webs or funnel formation. In the narrow depths of the cavity discharge poured from the mouth of the Eustachian tube.

9. A stitch in one case prevented healing till removed.

10. An island of bare bone existed in a few cases because granulations had failed to spring from it. A completely flat bed of granulations greatly enhances subsequent epithelialization.

\section{Technique of Operation}

The incision is prolonged downwards and forwards to the anterior aspect of the tip of the mastoid process. Then, when the membranous meatus is entirely detached, the whole auricle can be turned forwards by the retractor and the bony meatus wholly exposed with the mastoid process.

Many surgeons are at pains to open merely the antrum, and avoid, so far as possible, gouging the rest of the mastoid process. I believe this to be a mistake. If this method is followed the posterior meatal wall must be removed obliquely from behind forwards and inwards. The antrum then forms a posterior recess, and a narrow bony meatus remains. Superior results are obtained if the bone is hollowed out extensively, almost as low as the tip and as deep as the floor of the bony meatus. The whole of the posterior meatal wall is removed entirely, so that no facial ridge remains. Before the removal of the posterior meatal wall has been completed a flap is cut. Ballance's and Korner's flaps are admirable. The latter has the advantage that a small skin flap may be cut, bui a larger piece of cartilage removed. The conchal opening then slopes directly into the posterior reach of the cavity. It is quite large, but not unpleasantly obtrusive.

The field is now clear for completing the removal of the posterior wall of the meatus and the bridge. Shavings of bone are removed from below upwards, parallel with the facial canal, so that were the canal opened it would be recognized with its nerve intact. This method is more sure than relying upon the anaesthetist to watch the face. Between the elevation on the meatal floor and the lateral semicircular canal a slight ridge is unavoidably left, because here the nerve lies close beneath.

To avoid exposing the lateral sinus or dura of the middle fossa is a counsel of perfection. Many good surgeons try to follow it, and often fail. Such exposure appears to be of small consequence provided the dura is not damaged. Uncovered dura is resistant to infection, which, in chronic suppurative otitis media, is of low virulence ; proof of this is indicated by the healing of most radical incisions by first intention. It is certainly preferable to expose the dura rather than leave a plate of bone so slender that its vitality is in question. Organisms may penetrate it and thrive beneath, with the formation of an extradural or cerebral abscess. Granulations may fail to spring from it, and thus interfere with subsequent healing. If a tiny area of dura is exposed it is safer to prise up or gouge off its thin-shelved border of inner table. Here I may mention that I believe the use of burrs on the bone, and the application of bismuth-iodoform-paraffin paste, either of which blocks the crevices of the bone, and failure to gouge such fresh surfaces of bone as the meatal floor, may similarly be the causes of islets of bone failing to granulate and heal.

The remnant spike of outer epitympanic wall must be carefully gouged off. The elevation on the meatal flour should be lowered as a routine. This makes for easy access to the tympanum in after-treatment. Light curettage of the tympanic annulus and the remaining portion of the tympanum is necessary to get rid of any osteitis. Quite often a shallow osteitis here accounts entirely for the pathology of chronic suppuration, and has been sufficient to defy all efforts at conservative treatment. The hypotympanic area can be safely curetted. In sclerosed mastoids cells are non-existent here. With regard to the vexed question of curetting the Eustachian tube, I have not found that healing is more frequently assured by doing this. Naturally, in common with the rest of the tympanum, the orifice and commencement of the tube will be carefully curetted with a spoon. I do not believe in the existence of peritubal cells when the mastoid elsewhere is acellular.

Any of the faults in technique described may prevent healing. A Eustachian fistula is a natural sequel, and the tube is wrongly blamed. Apart from this, however, a fistula may exist after a carefully performed radical operation, and is the chief bugbear of the operation. Most bleeding points are dry when the artery forceps are removed, but a vessel in the flap usually requires ligature. Chromic gut should be avoided.

\section{After-treatment}

Aural surgeons differ in the after-treatment of mastoid cavities, some relying on cleansing with or without drops or powder, others packing with dry or medicated gauze. I have often observed that when at last the granulations have formed a pale, even, flat bed epithelialization very 
rapidly follows. Exuberant granulations delay or prevent the process. Anything that would be mildly astringent and absorb moisture should prepare quickly a favourable bed of granulation tissue and thus hasten the protess of healing. It should be soluble in the ear secretion, otherwise it will clog the cavity. Boric acid fulfils these requirements. A satisfactory routine is as follows:

After operation the cavity is packed lightly and completely with dry lodoform half-inch ribbon gauzo. This is removed in five or six days. Thereafter plain half-inch ribbon gauze, shaken out of sifted boric acid powder, is packed evenly throughout the cavity. This is repeated daily. Eventually, when there is little secretion and the lining is flat, simple mopping followed by insufflating a thin film of powder is all that is required.

\section{Modified Radical Opērations}

Modified radical operations are performed to remove localized disease and preserve the good hearing present. Such disease is usually located in either or all of the following sites: the postero-superior margin of the tympanic annulus, the attic, the aditus, and the antrum.

I am convinced that there is only one operation worth considering. I refer to that type which differs from a radical operation only in so far that the remains of the tympanic membrane, together with the malleus and incus, are left in position. The bridge and outer attic wall must be removed. A very large proportion of these cases heal and dry with full preservation of hearing. It is a great mistake to remove the incus; as this seriously curtails the hearing. If the hearing is good the incus may be presumed to be quite healthy, or only slightly diseased. Slight disease consists merely in loss of small parts of its substance, and is in no way comparable to the gross osteitis which may exist, for instance, in the outer wall of the aditus or epitympanum. If, therefore, these parts be removed the incus will become buried in healthy granulations, and will heal over satisfactorily.

If it be argued that the pre-operative hearing was poor, or that the incus was found to be grossly diseased and its removal justifiable, then a full radical operation should be carried out, as poor hearing is very seldom made worse by this procedure. It is essential to remove the bridge and outer attic wall, which are frequently diseased and, furthermore, act as a barrier to drainage and epithelialization, which can only occur when the underlying areas are thrown open to healthy granulation tissue. No advantage is sacrificed by removing the bridge, but a wet ear is the common penalty of leaving it.

The soundness of these principles is fully borne out by observing and comparing the results of various types of modified radical operations. Extracting the incus, or leaving the bridge, dissatisfies the patient by reducing hearing or failing to stop the discharge. In that type of conservative operation which I have described as being highly successful, this is only the case if the various pitfalls in technique described in the radical operation are as carefully avoided in this one. The after-treatment should be the same, and care should be taken to avoid damaging the drum membrane.

\section{LOWER SEGMENT GAESAREAN SECTION*} BY

JAMES RIDDELL, M.C., M.D., F.R.C.S.ED., M.C.O.G. HONORARY GYNAECOLOGICAL SURGEON, PRINCE OF WALES'S HOSPITAL, PLYMOUTH

One of the chief advantages of this operation is that an extended "trial of labour" can be allowed with less danger to the patient. The operation can be performed before labour begins or after labour has been in progress. There is no need for the hurry that there may be in the performance of the "classical " operation in which the breech is delivered first. The contractile part of the uterus is not interfered with, bleeding is less, and consequently there is less danger of shock. The main defence of the patient against sepsis-namely, the blood-is conserved, and that is the outstanding reason why there is less danger" of sepsis. The suture line lies tucked away low down, and is less likely to cause omentum or bowel to become adherent to it. Adhesions between the uterus and the abdominal wall are not likely to occur.

\section{Necessity for X-Ray Examination}

Radiographs should be taken in cases which are considered likely to require Caesarean section. It would be regrettable to perform a Caesarean operation only to deliver an anencephalic foetus. The radiograph, by showing a collapsed vault of the skull, would prevent this mistake from being made. The presence of twins, unsuspected before radiography, might weigh the scale against operation. A breech presentation in an elderly primigravida might decide the accoucheur in favour of operation, not only to save damage to the pelvic floor of the patient, but to minimize the risk of losing the life of the child.

\section{Operative Technique}

After the patient has been anaesthetized the bladder should be catheterized and the vagina lightly packed with gauze wrung out of dettol solution or Bonney's paint.

* Read at a meeting of the South-Western Branch of the British Medical Association at Newquay, November 19th, 1935.
A short subumbilical incision is made and small towels are clipped to the edges of the wound, after which the peritoneal cavity is opened and packed off to avoid soiling. It is not necessary to use a self-retaining retractor, which requires a longer incision for its employment. Small retractors are preferable, particularly if the bladder is inclined to bulge into the wound. With a clean knife a transverse incision is made in the lower uterine segment just above the reflection of the peritoneum off the bladder, if possible without opening the amniotic cavity. The incision is enlarged with scissors, laterally and slightly upwards, sufficiently to allow the head to be delivered. I have used Bonney's compressor and Willett's forceps to lessen the haemorrhage in the making of the incision, but find that they are not required, as bleeding is generally only slight. Willett's forceps are sometimes useful in assisting the birth of the head.

The fingers of the right hand inserted gently inside the lower edge of the incision in the uterine wall coax the head from the uterus, whilst the left hand is employed outside the abdomen in manipulating the breech to faclitate the birth of the head. I intend to make trial of one blade of Wrigley's forceps instead of the fingers of the right hand, although the fingers can appreciate the resistance of the tissues better than an instrument and are less likely to cause damage. But one blade of Wrigley's forceps is easier to control than one blade of a long forceps, and its use would obviate the insertion of the hand into the uterus, to which practice there might be a serious objection. Suturing is made easy by marking the ends of the uterine incision with tissue forceps whilst the incision is still being stretched by the shoulders, then the shoulders and the rest of the body are delivered.

\section{Delivery of the Placenta}

Posterior pituitary extract $1 \mathrm{c.cm}$. is injected into the uterine wall and the placenta and membranes are delivered by manipulating the fundus of the uterus with the left hand outside the abdomen. The placenta separates quickly, and there is no need to wait for it as may be necessary in natural delivery. It is essential to remove the membranes completely, using in some cases a small dry towel with which to peel them off the lower segment. The hand does not need to be introduced into the uterus in order to secure the placenta.

After the lower edge of the uterine incision has been carefully defined threo mattress sutures of hardened catgut are inserted to control haemorrhage. It is important to define 